\section{HÁBITOS DE VIDA SALUDABLES, GÉNERO Y CÁNCER}

\author{
Pedro Pérez-Segura \\ Hospital Clínico Universitario San Carlos \\ perezsegura09@gmail.com
}

\section{HEALTHY LIFESTYLES, GENDER AND CANCER}

Cómo citar este artículo/Citation: Pérez-Segura, P. (2015). "Hábitos de vida saludables, género y cáncer". Arbor, 191 (773): a232. doi: http://dx.doi.org/10.3989/arbor.2015.773n3002
Copyright: @ 2015 CSIC. Este es un artículo de acceso abierto distribuido bajo los términos de la licencia Creative Commons Attribution-Non Commercial (by-nc) Spain 3.0.

Recibido: 9 diciembre 2014. Aceptado: 24 abril 2015.

RESUMEN: Si tuviésemos que clasificar los factores de riesgo que nos hacen más proclives a desarrollar cáncer podríamos hacerlo, de una manera sencilla, en factores endógenos y exógenos. Dentro de los primeros estarían todos aquellos que tienen que ver con la herencia (genes de predisposición) o con alteraciones moleculares no hereditarias que hacen que nuestras células se "vuelvan" malignas con más facilidad, estos factores son inmodificables. Sin embargo, los factores exógenos dependen de los llamados "hábitos de vida", son factores modificables e influyen en un porcentaje importante de los cánceres más prevalentes en la actualidad, como son el de colon, mama y pulmón. Actuando sobre el tabaco y la obesidad se reduciría entre un $30 \%$ y $40 \%$ la incidencia de cáncer en los países industrializados. Este artículo es una revisión del papel de los hábitos de vida negativos en la producción del cáncer.

PALABRAS CLAVE: alcohol; factores reproductivos; obesidad; tabaco.
ABSTRACT: If we had to classify the risk factors making us more prone to develop cancer we could simply divide them into endogenous and exogenous factors. Among the endogenous factors we could find all those relating to heredity (genetic predisposition) or with non-hereditary molecular alterations that make our cells become malignant more easily. These factors are not modifiable; however, exogenous factors depend on lifestyle choices, which are. These factors have an influence on a large percentage of the most prevalent cancers nowadays, such as colon, breast and lung cancer. Taking action on tobacco and obesity would reduce the incidence of cancer by $30-40 \%$ in the industrialised countries. This article reviews the role of negative lifestyle choices in the development of cancer.

KEYWORDS: alcohol; reproductive factors; obesity; tobacco. 


\section{INTRODUCCIÓN}

Cada año se diagnostican en nuestro país 215.534 nuevos casos de cáncer (datos del año 2012), con una tasa de mortalidad de 102.762, para ese mismo año. Los avances producidos en los últimos años han permitido incrementar la supervivencia en la mayoría de los casos, pero la incidencia ha aumentado en algunos tumores, mayoritariamente aquellos que tienen que ver con hábitos de vida concretos.

Si tuviésemos que clasificar los factores de riesgo que nos hacen más proclives a desarrollar cáncer podríamos hacerlo, de una manera sencilla, en factores endógenos y exógenos. Dentro de los primeros estarían todos aquellos que tienen que ver con la herencia (genes de predisposición) o con alteraciones moleculares no hereditarias que hacen que nuestras células se "vuelvan" malignas con más facilidad; la características más importantes de estos factores son que son inmodificables y que influyen en un porcentaje pequeño de casos. Sin embargo, los factores exógenos serían aquellos que dependen de los llamados "hábitos de vida". En este caso, son factores modificables e influyen en un porcentaje importante de los cánceres más prevalentes en la actualidad (colon, mama, pulmón). Las cifras más creíbles indican que actuando sobre el tabaco y la obesidad se reduciría entre un $30 \%$ y $40 \%$ la incidencia de cáncer en los países industrializados.

Otro factor muy importante que debemos tener en cuenta cuando hablamos de cáncer es la previsión de lo que va a pasar en los próximos años; se espera que en el año 2030, la incidencia de cáncer en el mundo será de más de 21 millones de casos, lo que originará unos 13 millones de fallecimientos (Ferlay et al., 2010) (en la actualidad estamos hablando de unos 13 millones de casos al año con un total de 8 millones de fallecimientos). Este incremento de casos se debe, fundamentalmente, al aumento de la edad media de la población así como al aumento de casos en los países en desarrollo (en clara relación con la adherencia de hábitos de vida de los países industrializados), llegando casi a duplicar la incidencia actual en dichos países (McCormack y Boffetta, 2011).

En general, los hábitos de vida que más influyen en el riesgo de padecer cáncer son, para ambos sexos, el tabaco y la obesidad y, exclusivamente en las mujeres, los hábitos relacionados con aspectos hormonales. Repasaremos, en las próximas líneas, el papel que estos hábitos de vida negativos juegan en el desarrollo del cáncer.

\section{OBESIDAD}

Cuando se habla de obesidad la tendencia es pensar en patología cardiovascular como puede ser la hipertensión, diabetes o dislipemia; sin embargo, existe una relación muy estrecha entre ella y el cáncer, que analizaremos posteriormente. La forma de saber si una persona es obesa es mediante el cálculo del índice de masa corporal (IMC); se considera sobrepeso cuando oscila entre 25 y $29,9 \mathrm{~kg} / \mathrm{m}^{2}$ y obesidad cuando es $\geq 30 \mathrm{~kg} / \mathrm{m}^{2}$. Antes de nada, sería interesante ubicar el problema de la obesidad en nuestra sociedad. En los últimos años hemos asistido, quizá de manera silenciosa, al incremento del porcentaje de obesos en nuestro entorno: hemos pasado en nuestro país de un $7,7 \%$ de obesos en 1987 a un 13,6\% en el año 2001 (Salcedo et al., 2010). Y estas cifras son más alarmantes si nos centramos en los grados más extremos de la obesidad: incremento del 50\% para obesidades grado I, del $110 \%$ para las grado II y del $240 \%$ para las obesidades mórbidas (Basterra-Gortari et al., 2011). Además, en países como EEUU, un $17 \%$ de los niños y adolescentes son ya obesos (Ogden et al., 2010).

No hay duda de que se trata de un problema grave y creciente y que requiere medidas activas para luchar contra las complicaciones de esta enfermedad. Pero, ¿de qué manera se asocia la obesidad con el cáncer y viceversa? Existen diferentes trabajos que demuestran la clara relación existente entre obesidad y cáncer, fundamentalmente con los más prevalentes en los países industrializados. En el año 2002 la IARC (International Agency for the Research in Cancer) afirmó que algunos tipos de cáncer (colon, mama, riñón, endometrio y esófago) podían ser prevenidos si se reducía el sobrepeso. Posteriormente, un grupo de expertos mundiales aumentaron el número de cánceres comentado previamente, incluyendo el cáncer de mama en mujeres postmenopáusicas y también el de páncreas. Posteriormente han aparecido otros trabajos que, desde un punto de vista epidemiológico, llegan a las mismas conclusiones; quizá el más reciente es un estudio británico que analiza los 22 tumores más frecuentes en su entorno y demuestra una clara correlación entre 17 de ellos y el IMC así como las interacciones con otros factores de riesgo (por ejemplo, el tabaco) (Bhaskaran et al., 2014).

Sabemos, además, que los pacientes oncológicos obesos toleran peor los tratamientos antineoplásicos, tienen más complicaciones en su vida posterior tras finalizar dicho tratamiento y más posibilidades de desarrollar segundos tumores (Li et al., 2009; Mullen et al., 2008; Park et al., 2010). Desde el punto de vista 
metabólico, conocemos algunas alteraciones que conectan la obesidad con el cáncer (Calle y Kaaks, 2004); las dos más importantes son, quizá, la resistencia a la insulina y la hiperinsulinemia crónica posterior así como el aumento de la biodisponibilidad de las hormonas esteroideas y el proceso de inflamación crónica generado. Algunas de estas alteraciones reducen la apoptosis y activan la replicación celular. Existen dos sustancias enormemente interesantes en este proceso: por un lado, la adiponectina, que se relaciona inversamente con el IMC. Esta sustancia parece ejercer un cierto efecto antiinflamatorio y anticáncer. Por otro lado tenemos la leptina, que está asociada a la resistencia a la insulina.

Pero, ¿afecta por igual la obesidad a todos los tipos de cáncer? Veremos a continuación que no y la importancia que esto tiene para la mujer. Analizaremos, inicialmente aquellos tumores exclusivamente femeninos y el papel que la obesidad juega en su producción.

Un aspecto cada vez más estudiado es el de los disruptores endocrinos, sustancias químicas que por su pequeño tamaño tienen efectos hormonales en casi todos los seres vivos. La mayoría de los disruptores endocrinos funcionan a dosis muy pequeñas como las hormonas. Sus efectos pueden ser agonistas, bloquear la acción de las mismas, aumentar su síntesis o su metabolismo. Según el informe de la OMS, los pesticidas de uso actual se han relacionado en trabajadores expuestos a los mismos con el cáncer de mama, cáncer de próstata, cáncer de tiroides. Algunos disruptores, como los fenoles, pueden actuar sobre receptores estrogénicos, disminuyendo los niveles de adiponectina, aumentando la producción de insulina y disminuyendo la de glucagon, aumentando el estrés oxidativo y, como consecuencia, generando resistencia a la insulina, uno de los fenómenos clave en la relación obesidad-cáncer.

\subsection{Cáncer de mama}

Los datos disponibles en la actualidad señalan, de manera clara, la relación existente entre obesidad y cáncer de mama. Datos en población europea indican que más de un $16 \%$ de los cánceres de mama en mujeres postmenopáusicas están asociados a la obesidad (Calle y Kaaks, 2004). Sin embargo, está relación no es igual a lo largo de la vida de la mujer sino que el riesgo varía en función del estado hormonal de la misma (premenopáusicas vs postmenopáusicas). En el caso de las mujeres premenopáusicas parece existir un menor riesgo en aquellas que presentan un IMC $\geq 28 \mathrm{~kg} /$ $\mathrm{m}^{2}$ (Potischman et al., 1996), cosa que no ocurre en las mujeres postmenopáusicas, las cuales, presentan un incremento del riesgo del $30 \%$ si su IMC está entre 25 kg/m2 y 30 kg/m2 (Calle y Kaaks, 2004). Algunas de las razones que podrían explicar estas diferencias radicarían en que las mujeres premenopáusicas obesas presentan un mayor número de ciclos anovulatorios y menores niveles de hormonas esteroideas circulantes. Se ha intentado relacionar el cáncer de mama con el acúmulo a nivel abdominal; sin embargo, diferentes estudios han demostrado que si ajustamos el riesgo por el IMC de la población, el factor de la obesidad abdominal pierde su importancia (Harvie, Hooper y Howell, 2003). Otro aspecto de enorme importancia es la relación existente entre obesidad y evolución tumoral en mujeres obesas; diferentes trabajos han demostrado que se incrementa el riesgo de recaída en mujeres obesas así como una menor supervivencia (Rock y Demark-Wahnefried, 2002).

\subsection{Cáncer de endometrio}

Es este un cáncer en el que más evidencia hay del papel que juega la obesidad y el riesgo de desarrollar la enfermedad. Las personas que presentan sobrepeso y aquellas que son obesas presentan 2 y 3.5 veces más riesgo de desarrollar cáncer de endometrio, respectivamente. Algunos trabajos parecen demostrar que este riesgo es independiente del estado menopáusico y que el uso de terapia hormonal sustitutoria reduciría ese incremento de riesgo (McCullough et al., 2008). Para hacernos una idea del impacto de la obesidad en el riesgo de desarrollar cáncer de endometrio se calcula que el $45,2 \%$ de todos los cánceres de este tipo en población europea están en relación con la obesidad (Calle y Kaaks, 2004).

\subsection{Cáncer de ovario}

Los datos actuales no permiten relacionar la obesidad con un incremento del riesgo de desarrollar cáncer de ovario; de hecho, existen estudios con resultados contrarios en relación con el riesgo de desarrollar este tipo de cáncer en función del estado pre o postmenopaúsico (Lahmann et al., 2010; Schouten et al., 2008).

Existen otros cánceres, los cuales afectan tanto a hombres como a mujeres, que pueden tener una diferencia en la incidencia en función del sexo; veamos algunos datos interesantes al respecto.

\subsection{Cáncer colorrectal}

Sabemos que existe una relación clara entre obesidad y cáncer colorrectal. En el hombre el sobrepeso aumenta un $20 \%$ el riesgo de desarrollarlo, pero en 
las mujeres esta cifra se eleva hasta un $50 \%$. Se piensa que, en población europea, 1 de cada 4 casos de cáncer colorrectal en la mujer se debe a la obesidad (Calle y Kaaks, 2004). Sin embargo, datos del estudio EPIC han demostrado que el uso de terapia hormonal sustitutoria reduce el efecto que la obesidad tiene sobre este riesgo (Pischon et al., 2006).

\subsection{Cáncer de páncreas}

En este tumor no hay diferencias en cuanto al género del paciente, existiendo una clara relación entre sobrepeso/obesidad y el riesgo de desarrollar este tipo de cáncer. Datos publicados reflejan que si la persona tiene un IMC >30 presenta un $47 \%$ más de riesgo frente a aquellos sujetos cuyo IMC está entre 21 y 23 (Genkinger et al., 2011). Datos de nuestro entorno reflejan que el $19,3 \%$ de los cánceres de páncreas en población europea están en relación franca con la obesidad y el sobrepeso (Calle y Kaaks, 2004).

\subsection{Cáncer de riñón}

Al igual que en el cáncer de páncreas no existen diferencias por sexos pero la presencia de obesidad incrementa 2,5 veces el riesgo de desarrollar este tipo de tumores. En población europea se estima que casi uno de cada 3 cánceres renales tiene relación con la obesidad (Calle y Kaaks, 2004).

\subsection{Adenocarcinoma de esófago}

No existen datos que diferencien el riesgo de desarrollar este tipo de cáncer en función del género; lo que sí sabemos es que la incidencia de este tipo de neoplasia se ha incrementado mientras que se ha reducido la del carcinoma epidermoide de la misma localización, en relación clara con el avance de la obesidad en el mundo. Se estima que una persona obesa triplica el riesgo frente a una persona no obesa y que más del $42 \%$ de los casos de este tipo de neoplasia tienen relación con la obesidad y el sobrepeso (Calle y Kaaks, 2004).

\subsection{Cáncer de vesícula biliar y hepatocarcinoma}

Los datos son similares a los comentados anteriormente, con un incremento del riesgo del doble para el desarrollo de cáncer de vesícula biliar en los obesos (los datos para hepatocarcinoma indican cifras similares con un leve incremento en los hombres) (Calle y Kaaks, 2004).

\subsection{Cáncer de tiroides}

Tampoco parecen existir diferencias por género y parece existir un aumento del riesgo del $53 \%$ en aqueIlas personas obesas, especialmente para el subtipo papilar (Calle y Kaaks, 2004; Zhao et al., 2012).

Todos estos datos intentan mostrar el papel cada vez más importante que la obesidad tiene como factor de riesgo de desarrollar cáncer, con una especial atención en aquellos tumores exclusivamente femeninos. El incremento de la obesidad en la población mundial, asociado a una mayor longevidad y a la incorporación de las poblaciones de países en desarrollo a estos hábitos de vida auguran una auténtica epidemia en las próximas décadas, si no se toman medidas tanto desde el punto de vista de educación de la población como sociales para mejorar los hábitos dietéticos de la misma.

\section{TABACO}

La incorporación de la mujer al hábito tabáquico en las últimas décadas ha seguido una progresión continua. La reducción en la ratio de hombres/mujeres en la incidencia de cánceres claramente asociados al tabaco como factor de riesgo principal sirven para corroborar este dato. En el año 2010, la Organización Mundial de la Salud (OMS) arrojaba la tremenda cifra de que 200 millones de mujeres fumaban en el mundo Lejos de disminuir, esta cifra ha aumentado en los últimos años. Todos conocemos el impacto tan negativo que el tabaco tiene sobre la salud; enfermedades cardiovasculares, respiratorias y cáncer encabezan el listado de "beneficios" que esta droga tiene sobre la población, reduciendo la supervivencia de la población actual.

Cada año, el consumo de tabaco mata a más de 5 millones de personas en el mundo, y aproximadamente 1,5 millones de estas son mujeres. La mayoría de ellas (el 75\%) viven en países de ingresos bajos y medianos que se encuentran en desarrollo y que amenazan con incrementar estas cifras en los próximos años. De manera general, las mujeres fumadoras tienen más probabilidades de sufrir esterilidad y fumar durante el embarazo aumenta los riesgos de parto prematuro, muerte del recién nacido y puede disminuir la producción de leche materna.

La relación entre tabaco y cáncer es clara y conocida. El consumo de tabaco es responsable de casi 1 de cada 3 muertes por cáncer. Además de los cánceres asociados con más frecuencia por la población al tabaco (pulmón, laringe) es conveniente saber que 
otros cánceres, como el cáncer de cuello uterino, es 26 veces más frecuente en las mujeres fumadoras respecto a las que no fuman. En cuanto al cáncer de pulmón, cada año mata más mujeres que, por ejemplo, el cáncer de mama, siendo el tabaco el causante del 70\% de todos esos casos de cáncer de pulmón. El listado de cánceres cuyo riesgo se incrementa con el hábito de fumar es enorme: cánceres de cabeza y cuello, esófago, riñón, hígado, vejiga, páncreas, estómago, ovario, colorrectal y algunas neoplasias hematológicas (leucemias agudas).

\section{ALCOHOL}

El consumo de esta sustancia está claramente relacionado con la producción de cánceres de áreas tan variadas como cabeza y cuello, hígado, colorrecto y mama (Bagnardi et al., 2001; Cho et al., 2004; Hamajima et al., 2002). Se considera que, en el caso de la mujer, el riesgo de desarrollar cáncer de hígado puede ser mayor que en el hombre $(0,8 \%)$ y el cáncer de mama asociado a la ingesta de alcohol también se incrementa en un $0,8 \%$. El problema más importante del alcohol es la asociación, frecuente, de su consumo conjunto con el tabaco lo que produce una sinergia "mortal" para el desarrollo de estos cánceres.

Algunos países tienen datos reales sobre el papel que el alcohol juega como factor de riesgo para desarrollar cáncer. En el Reino Unido, por ejemplo, el alcohol es responsable del $11 \%$ de todos los cánceres de mama (lo que supone 5.000 tumores extra cada año); para determinar este incremento del riesgo, Naomi Allen y su equipo, de la Unidad de Epidemiología del Cáncer de la Universidad de Oxford, analizaron a 1.280.296 mujeres de mediana edad, que forman parte del 'Million Women Study' (Allen et al., 2009).

\section{FACTORES REPRODUCTIVOS}

Desde tiempos inmemoriales es conocido que hay una serie de factores reproductivos/hormonales que se asocian con un incremento en el riesgo de desarrollar cáncer de mama; entre ellos están el hecho de tener una menarquia precoz, una menopausia tardía, que el primer embarazo a término sea tardío, o la baja paridad. Se cree que el hecho de usar anticonceptivos orales o tratamientos hormonales sustitutorios también incrementan el riesgo respecto a las mujeres no usuarias de los mismos. Como factor protector podríamos señalar la lactancia materna, aunque sigue siendo una hipótesis no confirmada (Lacey et al., 2009).
Un tema de enorme interés en los últimos tiempos es el papel de la infección por el virus del papiloma humano (VPH) como factor de riesgo para desarrollar cáncer de cuello uterino (cérvix). Hay que aclarar que la mayoría de las infecciones por VPH de alto riesgo no causan cáncer. De hecho, muchas de ellas desaparecen en 1 ó 2 años. Sin embargo, las infecciones que duran muchos años aumentan el riesgo de una persona de presentar cáncer. Prácticamente todos los cánceres cervicales son causados por infecciones por VPH, y solo dos tipos, el 16 y el 18, son responsables de casi el $70 \%$ de todos los casos. Otros cánceres causados por este virus son el de ano, de vagina (casi la mitad son causados por este virus), de vulva y de pene (Muñoz et al., 2004; Parkin, 2006; Watson et al., 2008).

Este tipo de infecciones se transmite por vía sexual y es muy importante utilizar medidas que impidan el contagio, aunque los datos del uso de preservativos para evitar la infección no demuestran claramente su utilidad. En la actualidad, aquellas mujeres mayores de 12 años que no hayan estado en contacto con el VPH se pueden beneficiar de la vacunación, lo cual disminuye no solo la incidencia de cáncer de la zona sino también de infecciones secundarias a estos virus. Hay que recordar que el hecho de estar vacunada no exime a las mujeres de realizar de manera periódica las revisiones que sus médicos les recomienden por su grupo de riesgo concreto.

En conclusión, la relación existente entre género, cáncer y hábitos de vida es sólida y conocida. En la actualidad, tenemos factores claramente identificados y asociados a determinados tipos de cánceres. La proyección mundial del incremento de casos que se van a producir en los próximos años de cánceres asociados a determinados hábitos de vida obliga a replantearse las políticas preventivas y donde la mujer, además, debe jugar un papel fundamental. El incremento, por ejemplo, de mujeres fumadoras, incrementará de manera llamativa la incidencia de cánceres de pulmón o cérvix (aunque no sabemos el impacto que las políticas de vacunación contra el VPH pueden tener). Por otro lado, esperamos que las campañas de concienciación que se empiezan a realizar en temas tan importantes, por ejemplo, como la obesidad en conjunción con medidas diseñadas por los gobiernos para favorecer hábitos de vida saludables, tengan el impacto que todos esperamos y que ese $30 \%-40 \%$ de cánceres asociados a determinados hábitos de vida sea reducido a $0 \%$ en las próximas décadas. De nada vale investigar en el tratamiento del cáncer si no conseguimos cerrar el grifo del incremento de la incidencia 
del mismo; probablemente acabaremos convirtiendo esta enfermedad en un proceso crónico pero no conseguiremos nuestro objetivo final: eliminar el cáncer de nuestra sociedad.

\section{BIBLIOGRAFÍA}

Allen, N. E., Beral, V., Casabonne, D., Kan, S. W., Reeves, G. K., Brown, A. et al. (2009). Moderate alcohol intake and cancer incidence in women. Journal of the National Cancer Institute, 101, 5, pp. 296-305. DOI: djn514 [pii]10.1093/jnci/djn514.

Bagnardi, V., Blangiardo, M., La Vecchia, C. y Corrao, G. (2001). A meta-analysis of alcohol drinking and cancer risk. British Journal of Cancer, 85, 11, pp. 1700-1705. DOI: 10.1054/ bjoc.2001.2140S0007092001921408 [pii].

Basterra-Gortari, F. J., Beunza, J. J., Bes-Rastrollo, M., Toledo, E., García-López, M. y Martínez-González, M. A. (2011). Increasing trend in the prevalence of morbid obesity in Spain: from 1.8 to 6.1 per thousand in 14 years. Revista Española de Cardiología, 64, 5, pp. 424-426. DOI: S0300-8932(10)00095-3 [pii]10.1016/j. recesp.2010.06.010.

Bhaskaran, K., Douglas, I., Forbes, H., dosSantos-Silva, I., Leon, D. A. y Smeeth, L. (2014). Body-mass index and risk of 22 specific cancers: a population-based cohort study of 5.24 million UK adults. Lancet, 384, 9945, pp. 755-765. DOI: S0140-6736(14)60892-8 [pii]10.1016/ S0140-6736(14)60892-8.

Calle, E. E. y Kaaks, R. (2004). Overweight, obesity and cancer: epidemiological evidence and proposed mechanisms. $\mathrm{Na}$ ture Reviews Cancer, 4, 8, pp. 579-591. DOI: $10.1038 / n r c 1408$ nrc1408 [pii].

Cho, E., Smith-Warner, S. A., Ritz, J., van den Brandt, P. A., Colditz, G. A., Folsom, A. R. et al. (2004). Alcohol intake and colorectal cancer: a pooled analysis of 8 cohort studies. Annals of Internal Medicine, 140, 8, pp. 603-613. DOI: 140/8/603 [pii].

Ferlay, J., Shin, H. R., Bray, F., Forman, D., Mathers, C. y Parkin, D. M. (2010). Estimates of worldwide burden of cancer in 2008: GLOBOCAN 2008. International Journal of Cancer, 127, 12, pp. 28932917. DOI: 10.1002/ijc.25516.

Genkinger, J. M., Spiegelman, D., Anderson, K. E., Bernstein, L., van den Brandt, P. A., Calle, E. E. et al. (2011). A pooled analy- sis of 14 cohort studies of anthropometric factors and pancreatic cancer risk. International Journal of Cancer, 129, 7, pp. 1708-1717. DOI: 10.1002/ijc.25794.

Hamajima, N., Hirose, K., Tajima, K., Rohan, T., Calle, E. E., Heath, C. W., Jr, et al. (2002). Alcohol, tobacco and breast cancer--collaborative reanalysis of individual data from 53 epidemiological studies, including 58,515 women with breast cancer and 95,067 women without the disease. British Journal of Cancer, 87, 11, pp. 1234-1245. DOI: 10.1038/sj.bjc.6600596.

Harvie, M., Hooper, L. y Howell, A. H. (2003). Central obesity and breast cancer risk: a systematic review. Obesity Reviews, 4, 3, pp. 157-173.

Lacey, J. V., Jr., Kreimer, A. R., Buys, S. S., Marcus, P. M., Chang, S. C., Leitzmann, M. F. et al. (2009). Breast cancer epidemiology according to recognized breast cancer risk factors in the Prostate, Lung, Colorectal and Ovarian (PLCO) Cancer Screening Trial Cohort. BMC Cancer, 9, pp. 84. DOI: 14712407-9-84 [pii]10.1186/1471-2407-9-84.

Lahmann, P. H., Cust, A. E., Friedenreich, C. M., Schulz, M., Lukanova, A., Kaaks, R. et al. (2010). Anthropometric measures and epithelial ovarian cancer risk in the European Prospective Investigation into Cancer and Nutrition. International Journal of Cancer, 126, 10, pp. 24042415. DOI: 10.1002/ijc.24952.

Li, D., Morris, J. S., Liu, J., Hassan, M. M., Day, R. S., Bondy, M. L. et al. (2009). Body mass index and risk, age of onset, and survival in patients with pancreatic cancer. Journal of the American Association, 301, 24, pp. 2553-2562. DOI: 301/24/2553 [pii]10.1001/jama.2009.886.

McCormack, V. A. y Boffetta, P. (2011). Today's lifestyles, tomorrow's cancers: trends in lifestyle risk factors for cancer in lowand middle-income countries. Annals of Oncology, 22, 11, pp. 2349-2357. DOI: mdq763 [pii]10.1093/annonc/mdq763.

McCullough, M. L., Patel, A. V., Patel, R., Rodriguez, C., Feigelson, H. S., Bandera, E. V. et al. (2008). Body mass and en- dometrial cancer risk by hormone replacement therapy and cancer subtype. Cancer Epidemiology, Biomarkers and Prevention, 17, 1, pp. 73-79. DOI: 10559965.EPI-07-2567 [pii]10.1158/10559965.EPI-07-2567.

Mullen, J. T., Davenport, D. L., Hutter, M. M., Hosokawa, P. W., Henderson, W. G., Khuri, S. F. et al. (2008). Impact of body mass index on perioperative outcomes in patients undergoing major intra-abdominal cancer surgery. Annals of Surgical Oncology, 15, 8, pp. 2164-2172. DOI: 10.1245/s10434-008-9990-2.

Muñoz, N., Bosch, F. X., Castellsague, X., Diaz, M., de Sanjose, S., Hammouda, D. et al. (2004). Against which human papillomavirus types shall we vaccinate and screen? The international perspective. International Journal of Cancer, 111, 2, pp. 278-285. DOI: 10.1002/ijc.20244.

Ogden, C. L., Carroll, M. D., Curtin, L. R., Lamb, M. M. y Flegal, K. M. (2010). Prevalence of high body mass index in US children and adolescents, 2007-2008. Journal of the American Association, 303, 3, pp. 242-249. DOI: 2009.2012 [pii]10.1001/jama.2009.2012.

Park, J. S., Choi, G. S., Jang, Y. S., Jun, S. H. y Kang, H. (2010). Influence of obesity on the serum carcinoembryonic antigen value in patients with colorectal cancer. Cancer Epidemiology, Biomarkers and Prevention, 19, 10, pp. 24612468. DOI: 1055-9965.EPI-10-0569 [pii]10.1158/1055-9965.EPI-10-0569.

Parkin, D. M. (2006). The global health burden of infection-associated cancers in the year 2002. International Journal of Cancer, 118, 12, pp. 3030-3044. DOI: 10.1002/ijc. 21731.

Pischon, T., Lahmann, P. H., Boeing, H., Friedenreich, C., Norat, T., Tjonneland, A., et al. (2006). Body size and risk of colon and rectal cancer in the European Prospective Investigation Into Cancer and Nutrition (EPIC). Journal of the National Cancer Institute, 98, 13, pp. 920-931. DOI: 98/13/920 [pii]10.1093/jnci/djj246. 
Potischman, N., Swanson, C. A., Siiteri, P. y Hoover, R. N. (1996). Reversal of relation between body mass and endogenous estrogen concentrations with menopausal status. Journal of the National Cancer Institute, 88, 11, pp. 756-758.

Rock, C. L. y Demark-Wahnefried, W. (2002). Nutrition and survival after the diagnosis of breast cancer: a review of the evidence. Journal of Clinical Oncology, 20, 15, pp. 3302-3316.

Salcedo, V., Gutierrez-Fisac, J. L., GuallarCastillon, P. y Rodriguez-Artalejo, F. (2010). Trends in overweight and misperceived overweight in Spain from 1987 to 2007. International Journal of Obesity, 34, 12, pp. 1759-1765. DOI: ijo201096 [pii]10.1038/ijo.2010.96.

Schouten, L. J., Rivera, C., Hunter, D. J., Spiegelman, D., Adami, H. O., Arslan, A. et al. (2008). Height, body mass index, and ovarian cancer: a pooled analysis of 12 cohort studies. Cancer Epidemiology, Biomarkers and Prevention, 17, 4, pp. 902-912. DOI: 1055-9965.EPI-07-2524 [pii]10.1158/1055-9965.EPI-07-2524.

Watson, M., Saraiya, M., Ahmed, F., Cardinez, C. J., Reichman, M. E., Weir, H. K. et al. (2008). Using population-based cancer registry data to assess the burden of human papillomavirus-associated cancers in the United States: overview of methods. Cancer, 113, 10 Suppl, pp. 2841-2854. DOI: 10.1002/cncr. 23758.

Zhao, Z. G., Guo, X. G., Ba, C. X., Wang, W., Yang, Y. Y., Wang, J. et al. (2012). Overweight, obesity and thyroid cancer risk: a meta-analysis of cohort studies. Journal of International Medical Research, 40, 6, pp. 2041-2050. 